\title{
Evaluation of Large Scale Online Maps
}

\author{
Michael P. Peterson ${ }^{\mathrm{a}, *}$ \\ ${ }^{a}$ University of Nebraska at Omaha, Omaha, Nebraska, USA \\ * Corresponding author
}

Keywords: Multi-Scale Pannable (MSP) maps, Online Mapping Services, Tile-based Mapping, Large-Scale Maps

Abstract:

Large scale, zoomed-in maps as available from Google Maps, Microsoft Bing Maps, MapBox, HERE, Apple and OpenStreetMap and other online map services are one of their primary benefits. Maps at this scale are not available to most individuals from any other source. The features and labels that are included on these large-scale maps are an important indicator of the completeness of the underlying database and the quality of map rendering.

Online mapping services all rely on a database of vectors composed of points, lines, and areas along with feature attributes. When using these services, we view a rendered version of the vector data in multiple scales. The rendering process involves the scale-dependent generalization and symbolization of the spatial data. Rendered map tiles can then be zoomed and panned from side-to-side producing what we refer to as multi-scale pannable (MSP) maps.

Since the introduction of the technique in 2005 by Google, all major online map providers have adopted the same underlying technology. Vector data is projected and divided into vector tiles at multiple scales. The tile boundaries are identical between all mapping services. The maps vary only as a result of differences in the underlying vector database, and the generalization / symbolization used in their rendering.

Comparing maps from these services helps to evaluate both the quality of the underlying spatial data and the rendering process (see Figure 1). A comparison procedure is used that generates large scale map pairs at random locations using the Application Programmer Interface (API) for three different mapping services. The quality of each representation is then evaluated for feature and label density. The comparison is done for three different continents. For North America, it was found that maps from Google had consistently higher feature and label density than those from Microsoft Bing and Mapbox. Google Maps also held an advantage in Europe. Maps from Microsoft Bing were more detailed in Sub-Saharan Africa compared to both Google Maps and Mapbox. Maps from Mapbox, relying exclusively on data from OpenStreetMap, had the lowest feature and label density for all three areas.

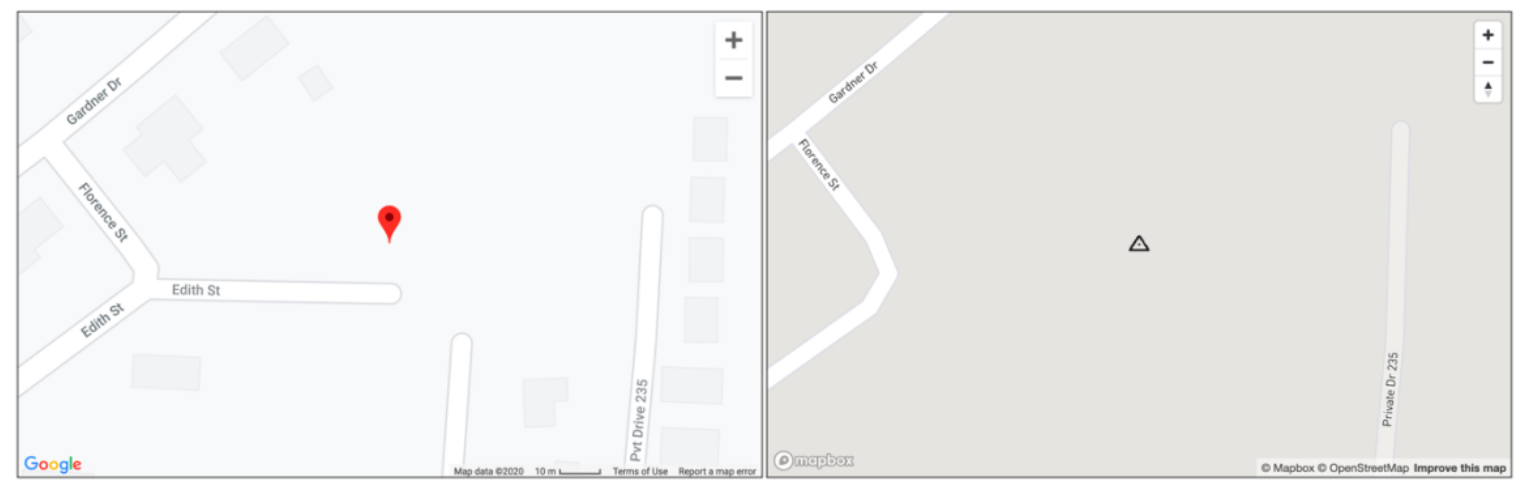

Figure 1. A side-by-side comparison between Google Maps and Mapbox for a random location in North America.

The pairwise comparison procedure used here can be applied in numerous other ways. For example, an assessment could be made as to which map has more legible text, better design elements, more appropriate generalization for the particular scale, or is even drawn more quickly. Other map providers could also be evaluated such as MapQuest, Here.com, Apple and ESRI. The comparisons could also be done for different parts of the world such as Asia, Australia or South America. A more automated approach could also be implemented, perhaps implementing optical character recognition (OCR) for the recognition of text. The methodology presented here opens many avenues for future research to evaluate the quality of MSP maps presented by online services. 